\title{
Retinal burns caused by exposure to MIG-welding arcs: report of two cases
}

\author{
G P H BRITTAIN
}

SUMMARY A new generation of arc welder has recently become widely available at a price which is within reach of most amateurs and part-time mechanics, known as the MIG welder (metal-arc inert gas welder). In MIG welding the arc is ensheathed in a stream of inert gas which prevents the molten metal from oxidising. The stream of gas changes the character of the emitted radiation, and it is possible that this type of welder poses a greater threat to sight than previously recognised. Radiation in the ultraviolet range emitted by arc welders is absorbed by the unprotected cornea and lens, giving rise to a keratoconjunctivitis, or 'arc-eye,' which, though intensely painful, is not considered a threat to sight. Radiation in the visible and near infrared spectrum, however, penetrates the eye to be absorbed by the retina and may cause thermal or photochemical damage which may be permanent and sight-threatening. Retinal injuries resulting from exposure to ordinary electric welding arcs have been reported, but such injuries are uncommon. Two cases of retinal burns resulting from exposure to MIG welder emissions which presented on consecutive days to the Leicester Royal Infirmary are presented. This is the first report of such injuries relating specifically to MIG welding.

The radiation emitted from electric welding arcs can extend across a wide spectrum from infrared to ultraviolet and beyond. ${ }^{2}$ Radiation in the ultraviolet range emitted by arc welders is absorbed by the unprotected cornea and lens, giving rise to keratoconjuntivitis, 'welder's flash,' or 'arc-eye,' a condition known to welders and accepted as an occupational hazard which, although intensely painful, is not considered a threat to sight in the long term. But radiation in the visible and near infrared spectrum $(400-1400 \mathrm{~nm})$ penetrates the eye, to be absorbed by the retina, ${ }^{3}$ where, given sufficient intensity and duration, it will cause thermal or photochemical damage $^{\text {t. }}$ which may be permanent and sight threatening. Retinal injuries resulting from exposure to welding arc radiation have been reported, ${ }^{7-9}$ but such injuries are uncommon.

A new generation of arc welder has recently become widely available at a price which is within the reach of most amateurs and part-time mechanics, known as the MIG welder (metal-arc inert gas welder) or GMAW (gas metal arc welder) in the USA. In MIG welding the arc is ensheathed in a stream of inert gas which prevents the molten metal

Correspondence to Mr G P H Brittain FRCS, Department of Ophthalmology, Leicester Royal Infirmary, Leicester LE1 5WW. from oxidising in air. ${ }^{2}$ The stream of inert gas changes the character of the emitted radiation, and it is possible that this type of welder poses a greater threat to sight than previously recognised.

Two cases of retinal burns resulting from exposure to MIG welder emissions which presented on consecutive days to the Leicester Royal Infirmary are presented. This is the first report of such injuries relating specifically to MIG welding.

\section{Case reports}

CASE 1

A 26-year-old unemployed mechanic with no previous arc welding experience borrowed a MIG welder to repair the bodywork of his car. The protective visor was not used because outside lighting conditions were poor, making it difficult to see through the glass filter. The patient estimated that he continued to weld intermittently over the course of one and a half hours with no eye protection. The only attempt to protect the eyes was made by 'looking away from the arc' and holding the welder at arm's length. The car's mild steel panels were welded with $0.6 \mathrm{~mm}$ coppered steel wire to form the arc and Brewer's carbon dioxide as the inert gas.

Later, in the dark, he became aware of a persistent 
Right Eye

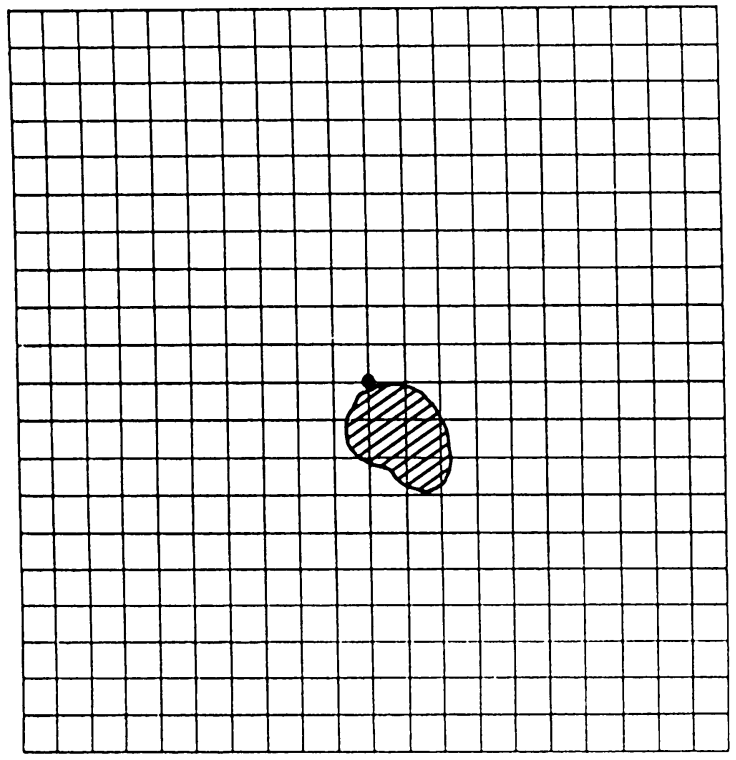

Left Eye

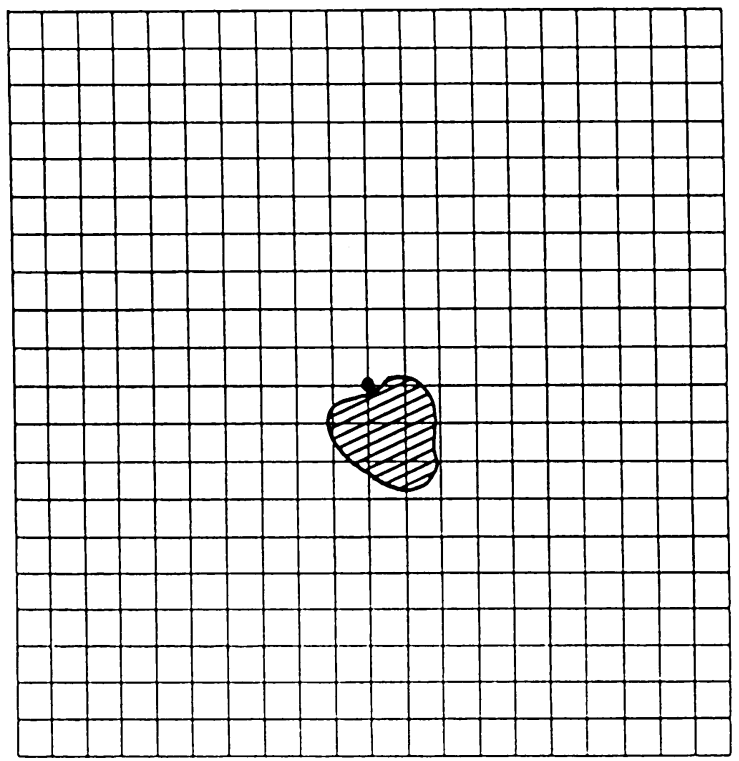

in right and lefteyes.

central field in both eyes. This was plotted by the patient on an Amsler grid at $30 \mathrm{~cm}$ (Fig. 1). A slight gritty sensation in both eyes was admitted to only on direct questioning. Unaided visual acuity was
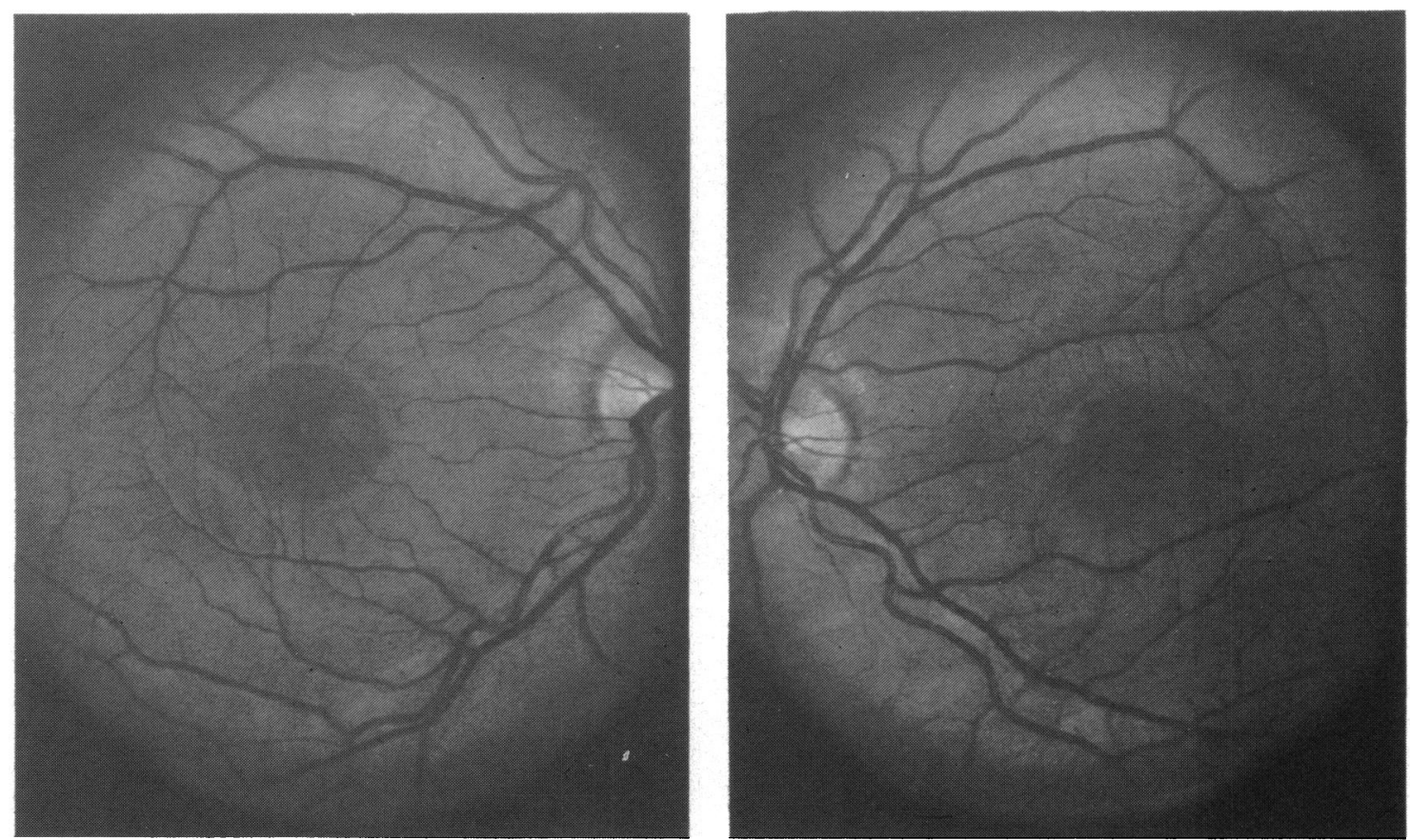

Fig. 2 Smalldarklesionabove each fovea. L: righteye. R: lefteye.

bright spot near the centre of his vision. He presented to his general practitioner and was referred on to hospital the following morning, still complaining of a positive scotoma affecting the lower part of his 
recorded as $6 / 5$ in both eyes. Minimal, punctate corneal fluorescein staining was seen on each eye. Retinal examination revealed a triangular, oedematous lesion approximately $0.1 \mathrm{~mm}$ in size just above and to the patient's left of each fovea (Fig. 2).

One week later the patient's condition was reviewed. At that time he was symptom free and the retinal lesions were no longer visible. Octopus perimetery (program 34) and fluorescein angiograms of both fundi were normal.

\section{CASE 2}

A 39-year-old Asian self-employed motor mechanic presented to the hospital casualty department in the early hours of the morning complaining of painful red eyes, photophobia, and blurred vision. The previous afternoon he had been welding a car body with a MIG welder. It was used underneath the car in poor light, where it was not possible to see through the darkened glass of the protective visor. The patient admitted to 'occasionally' looking round the side of the visor 'for never more than a few seconds' until the arc was struck; enough light would then be produced to enable him to see through the glass filter and allow the visor to be used in the correct manner. The inert gas used was a mixture of argon $75 \%$ and carbon dioxide $25 \%$. The arc was formed with $0.6 \mathrm{~mm}$ coppered steel wire, and the car body parts were of mild steel. He was inexperienced at welding and had used the machine five or six times, with a total welding time of less than five hours in the previous

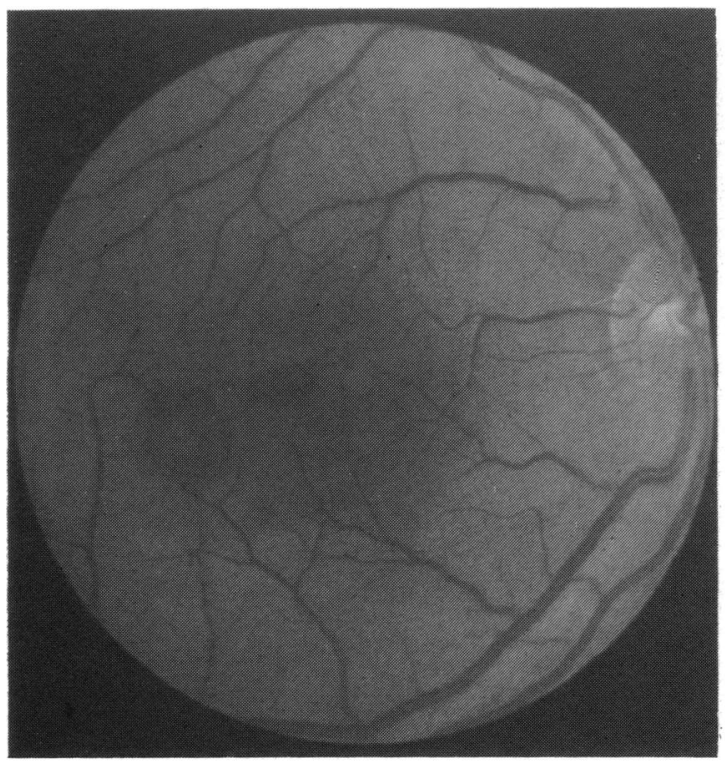

Fig. 3 Retinal burnsin botheyes. L: right eye. R: lefteye. seven months. He had never received any formal welding training nor had he done any other arc welding.

He was treated with topical antibiotic drops and eye padding. Seen in the eye department later that morning he complained of blurred vision and dark spots in both eyes; the eyes were still painful and photophobic. His corrected visual acuity was $6 / 7.5$ in the right and $6 / 9$ in the left eye. His conjunctivas were hyperaemic, and on slit-lamp examination both corneas showed fine, superficial, punctate fluorescein staining in the areas exposed by the palpebral fissures. Facial ultraviolet erythema was not noted. Examination of the fundi revealed two discrete circular brown coloured lesions in each eye. The lesions were located rather symmetrically temporal to either fovea. The larger was over half a disc diameter in size, the other a third of a disc diameter in size in both eyes; each lesion contained a concentric ring. The smaller lesion in the left eye was less marked than the other lesions (Fig. 3).

One week later the patient was symptom free and his corrected visual acuity had improved to $6 / 6$ in both eyes. Fundus fluorescein angiograms revealed hyperfluorescent areas in the early arterial phase without any leakage of fluorescein in later pictures (Fig. 4). These areas had the appearance of window defects in the pigment epithelium in locations corresponding to the retinal lesions, and looked like those found in retinal photocoagulation burns. ${ }^{10}$ No visual field defect could be located despite a careful

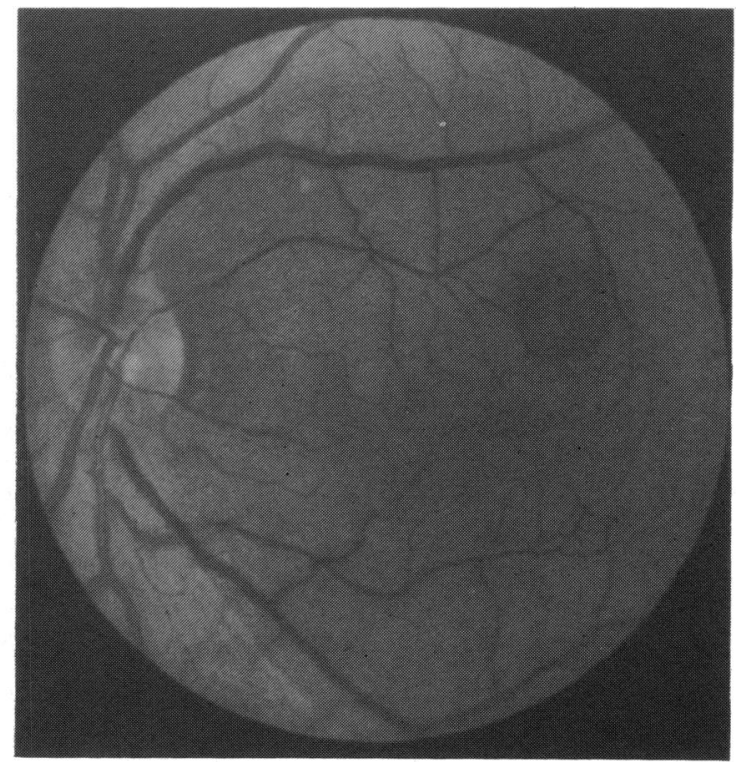


and detailed search with both tangent screen and computerised Octopus central field analyser (programs 34 and 64).

\section{Discussion}

Traditionally, the primary consideration of the ocular hazards of arc welding has been keratoconjunctivitis caused by the ultraviolet radiation, which is produced in large amounts by the welding arc. Ultraviolet radiation is absorbed by the cornea and lens ${ }^{3}$ and therefore does not pose a threat to the retina in the phakic eye. However, arc welding emissions cover a wide spectrum, much of which falls within the visible range. ${ }^{\prime}$ The danger of excessive retinal exposure to 'blue light' is well recognised. ${ }^{4-611}$

The first case demonstrates a macular burn resulting from prolonged exposure to the radiation of a carbon dioxide MIG welding arc. Although a full recovery was made, the injury was significant, for it shows that a sufficient proportion of the radiation produced by the arc is within the spectral range that can reach the retina to cause retinal injury. The lack of a marked keratoconjunctivitis despite the retinal involvement is of interest and may be explained by spectral analysis of the carbon dioxide arc which produces relatively less radiation in the ultraviolet range than in the visible and near infra-red spectrum (Fig. 5). ${ }^{12}$

The macular burns in the second case, which resulted from exposure to radiation from an argon

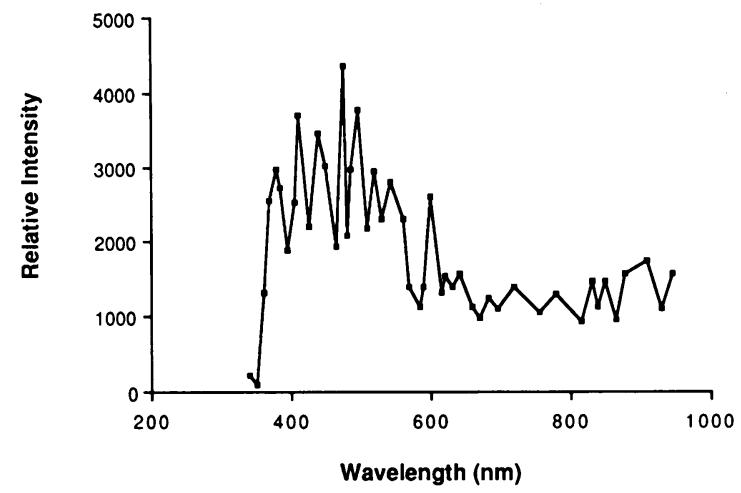

Fig.5 Emissionspectrum of carbon dioxidearc.

arc, were symmetrically located temporal to the fovea. The principle of physiological diplopia ${ }^{13}$ is well illustrated, showing that the welding arc must have been closer to the eyes than the point of visual fixation (Fig. 6). As the intensity of light incident on the retina is inversely proportional to the square of the distance of the source from the eye, this evidence that the patient occasionally had his face very close to the arc is a major factor in this retinal injury. 114

The total amount of radiation from an arc in any given period is approximately proportional to the square of the current and directly related to the arc length (or arc voltage). When using comparable nonprofessional equipment MIG welders operate at similar voltages but at almost twice the current of rod
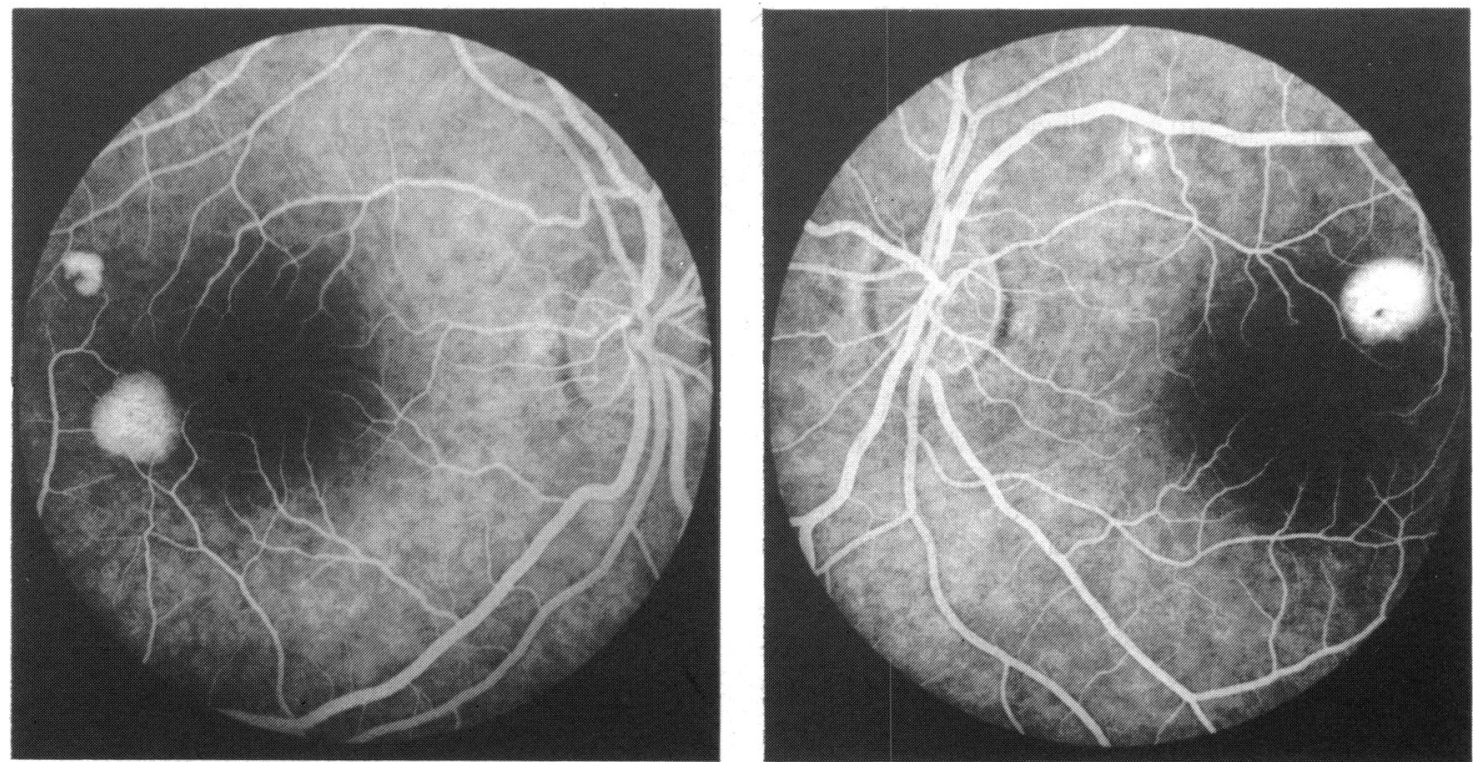

Fig. 4 Fundusfluorescein angiograms of both eyes showing hyperfluorescent areas at thesites of the retinal burns. 


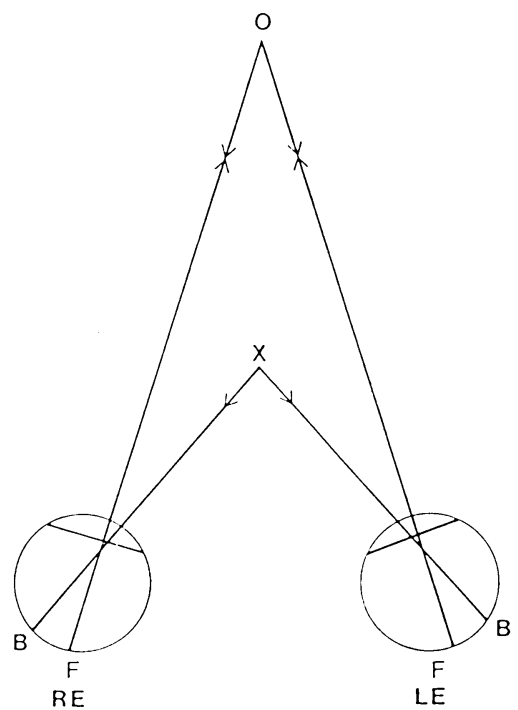

Fig. 6 Illustration of the principle of physiological diplopia. $F=$ fovea, $X=$ welding arc, $B=$ retinal burn, $O=$ fixation object.

arc welders. Whereas a welding rod is of some $2 \mathrm{~mm}$ in diameter and produces an arc about $2.5 \mathrm{~mm}$ in diameter, the MIG uses a wire $0.6 \mathrm{~mm}$ in diameter producing an arc less than $1 \mathrm{~mm}$ in diameter. At similar operating currents a MIG produces a current density six times higher (or six times brighter) than a rod arc. MIG welding differs from standard rod arc welding in that the molten metal is protected from oxidation in air by a stream of inert gas rather than by a melted flux. Radiation from a MIG welding arc is not blocked by the surrounding fumes and the molten slag in which a conventional rod arc is submerged. The MIG welding arc is produced by direct current, and its emissions are continuous. A traditional rod arc is driven by an alternating current and so the emissions are pulsed and interrupted, reducing the total exposure for a given period. These factors mean that a MIG arc is many times more intense than a conventional arc (Salter GR, personal communication).

The stream of inert gas in a MIG welder allows a controlled, steady arc to be formed. Since every substance has its characteristic ionisation emission spectra, introducing an inert gas to an electric arc will change the spectrum accordingly. When argon is excited by an electrical discharge, the majority of its most intense spectral bands fall within the visible and near infrared range ${ }^{15}$ (Fig. 7). The combined emission spectra of argon and carbon dioxide from the gas mix used by MIG welding machines is maximal across the visible spectrum. ${ }^{12}$ Such light freely traverses the ocular media (Fig. 8) to be absorbed by the retinal

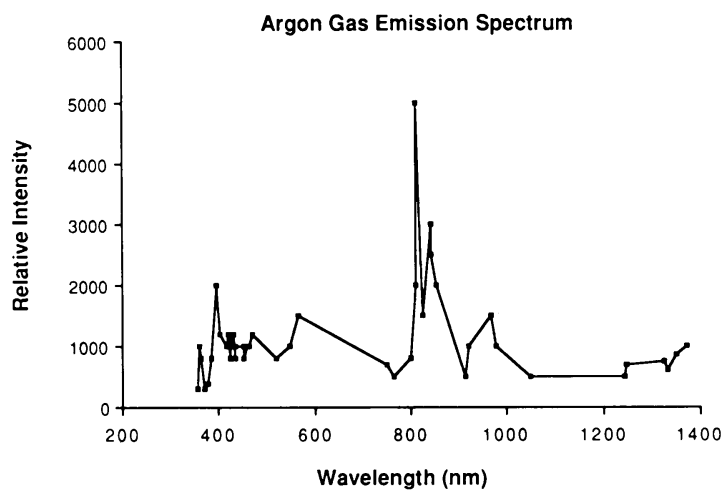

Fig. 7 Argongasemissionspectrum showing the relative intensity of the major emission bands.

pigment epithelium and choroid ${ }^{211+4}$ - the basis of argon laser retinal therapy.

Time-resolved optical spectra from 200 amp MIG welding arc ignitions have been analysed ${ }^{16}$ and show that there is surge of radiation in the ultraviolet and blue light range that occurs within the first 50 milliseconds of the arc being struck. The wave length of the emitted radiation is inversely proportional to the current driving the $\operatorname{arc}^{2}$ (Salter GR, personal communication) and it is likely that, at the lower currents at which amateur MIG welding machines operate (below 100A), more of the emission will be of a longer wavelength and able to reach the retina during this initial surge on striking the arc.

The filter recommended by the British Standards Protection of Eyes Regulations 1974 for MIG welding is considerably darker than that recommended for rod arc welding, making it more difficult to see through before the arc is struck. The temptation for the inexperienced welder to look round the visor to see

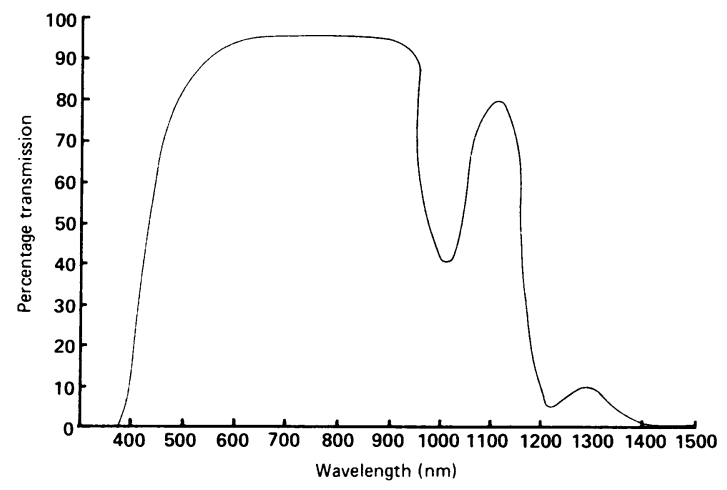

Fig. 8 Percentageemission throughocularmedia. Spectral transmission curve of ocularmedia (after Geeraets and Berry ${ }^{3}$ ). 
where the arc will ignite is understandable, especially in poor background lighting conditions where it is impossible to see through the visor. In these conditions pupils would be dilated, allowing a much higher part of the incident light to be focused on the retina. The amount of light falling on the retina is proportional to the square of the pupillary diameter. ${ }^{13}$ Pupillary constriction in response to striking the arc is too slow to block the initial $50 \mathrm{~ms}$ surge of radiation.

Safety advice given with amateur welding equipment does not adequately stress the risk of not wearing eye protection at all times, the importance of good background lighting, or the danger of welding too close to the eyes. ${ }^{17}$

MIG welding machines were at one time expensive and accessible only to the trained professional. In the past few years they have become readily available at a price within easy reach of the average amateur. They can be worked from a standard 13 amp domestic electricity power supply, and, unlike standard arc welding techniques, MIG welding requires very little skill to use and is ideally suited for welding the thin sheet metal on cars. ${ }^{17}$

The intensity and spectrum of radiation of MIG welders differ from those of standard rod arc welders and may present a more serious risk to sight in the unprotected eye than previously recognised, especially in the inexperienced hands of the amateur. It is likely that in future, with the increasing popularity of MIG welding machines, their misuse will give rise to many more cases of macular burns.

I am greatly indebted to Dr George R Salter, of the Welding Institute, Abington, Cambridge, for his help in supplying much of the technical information.

\section{References}

1 Naidoff MA, Sliney DH. Retinal injury from a welding arc. $A m J$ Ophthalmol 1974; 77: 663-8.

2 Koenigsberger F, Adair JR. Welding technology 3rd ed. London: Macmillan, 1965

3 Geeraets WJ, Berry ER. Ocular spectral characteristics as related to hazards from lasers and other sources. Am J Ophthalmol 1968; 66: $15-20$.

4 Mainster MA, Ham WT, Delori FC. Potential retinal hazards. Ophthalmology 1983; 90: 927.

5 McDonald HR, Irvine AR. Light-induced maculopathy from the operating microscope in extracapsular cateract extraction and intraocular lens implantation. Ophthalmology 1983; 90: 945-51.

6 Robertson DM, Feldman RB. Photic retinopathy from the operating room microscope. Am J Ophthalmol 1985; 101: 561-9.

7 Uniat L, Olk RJ, Hanish SJ. Arc welding maculopathy. Am J Ophthalmol 1986; 102: 394-5.

8 Minton JH. Occupational diseases of the lens and retina. Br Med J 1949 ; i: 392.

9 Wurdemann HV. The formation of a hole in the macula. Light burn from exposure to electric welding. Am J Ophthalmol 1936; 19:457.

10 Zweng HC, Flocks M, Peabody R. Histology of human ocular laser coagulation. Arch Ophthalmol 1966; 76: 11-5.

11 Sliney DH. Eye protective techniques for bright light. Ophthalmology. 1983; 90: 937-44.

12 Inoue K. Image processing for on-line detection of welding process (Report III) - improvement of image quality by incorporation of arc. Transactions of the Japanese Welding Research Institute. 1981; 10: 13-8.

13 Roper-Hall G, Burde RM. In: Moses RA, ed. Adler's physiology of the eye. St Louis: 1981; 7: 175.

14 Sliney DH, Freasier BC. Evaluation of optical radiation hazards. Appl Optics 1973; 12: 1-24.

15 Striganov AR, Sventitskii NS. Table of spectral lines of neutral and ionised atoms. Atomic spectroscopy laboratory IV Kurchatov laboratory of atomic energy. New York: IFI/Plenum Press, 1968.

16 Eriksen P. Time resolved optical spectra from MIG welding arc ignitions. Am Ind Hyg Assoc J 1985; 46: 101-4.

17 Porter L. MIG welding step by step. SIP (Industrial Products) Ltd, Loughborough: 1984.

Accepted for publication 28 April 1988. 